\title{
DESENVOLVIMENTO DE UM CÓDIGO PARA A ESTIMATIVA DA COMBINAÇÃO IDEAL DE MATERIAIS OBTURANTES ADICIONADOS AOS FLUIDOS DE PERFURAÇÃO
}

\author{
B. A. MOREIRA ${ }^{1 *}$, J. J. R. DAMASCENO ${ }^{2}$, J. R. GUIMARÃES ${ }^{3}$ \\ ${ }^{1}$ Universidade Tecnológica Federal do Paraná, Departamento Acadêmico de Engenharia Química \\ ${ }^{2}$ Universidade Federal de Uberlândia, Faculdade de Engenharia Química \\ ${ }^{3}$ Universidade Tecnológica Federal do Paraná, Departamento Acadêmico de Física, Estatística e \\ Matemática \\ *e-mail: brunomoreira@utfpr.edu.br
}

\begin{abstract}
RESUMO
Durante a perfuração de um poço de petróleo, busca-se a obstrução rápida e precisa dos poros da formação para reduzir o influxo do fluido de perfuração para o interior do reservatório. A combinação otimizada de materiais que fornece a granulometria ideal dos materiais obturantes auxilia na obstrução dos poros formando um reboco fino e de baixa permeabilidade. Neste contexto, este trabalho desenvolveu um código para a determinação da combinação ideal dos obturantes. A modelagem foi baseada em métodos de inversão que minimizam as diferenças entre a distribuição de partículas obtida pela combinação de materiais e a distribuição ideal proposta por modelos de empacotamento ideal existentes na literatura. Os resultados mostraram que a ferramenta desenvolvida pode ser utilizada para obtenção da melhor combinação de materiais, sendo portanto indicada para auxiliar na resolução de problemas práticos associados a formação do reboco.
\end{abstract}

\section{INTRODUÇÃO}

Uma função importante que os fluidos de perfuração exercem durante a etapa de perfuração de poços em rochas-reservatório é a formação do reboco. Nos casos em que os fluidos de perfuração são dotados de materiais obturantes, o influxo da parte líquida do fluido de perfuração (filtrado) para o interior da formação ocasiona a formação de uma torta de filtração comumente chamada de reboco. $\mathrm{O}$ reboco tem como principal objetivo, reduzir o influxo de filtrado no reservatório (MOREIRA, 2014; WALDMANN, 2005).

Desta forma, para a obstrução rápida e precisa dos poros da rocha e a formação de um reboco externo fino e de baixa permeabilidade, torna-se necessário estimar a concentração ideal e a melhor faixa granulométrica dos materiais obturantes no fluido de perfuração.

Em 1977, Abrams mencionou o tamanho médio das partículas dos obturantes devem ser igual ou ligeiramente maior do que $1 / 3$ do diâmetro médio de poros da formação rochosa.

Dick et al (2000), definiram a teoria do empacotamento ideal (TEI). A TEI consiste em uma aproximação gráfica para determinar a distribuição granulométrica otimizada dos obturantes para uma determinada formação rochosa. Tal técnica baseia-se no maior tamanho de poros dominante da formação ou fratura e na regra conhecida como $\mathrm{D}^{1 / 2}$, proposta por Kaeuffer, 1973.

Em 2007, Wenqiang e Jienian complementaram a regra de Dick et al (2000) ao desenvolverem um software para a determinação da proporção em que três 
materiais obturantes devem ser combinados para formar um reboco com a menor permeabilidade possível. Para isso, os autores basearam-se nas modelagens matemáticas de McGreay (teoria do empacotamento) e de Fuller.

Enbaia Dr e Ramdzani (2014) ao analisarem imagens, concluíram que a permeabilidade da formação rochosa não deve ser um parâmetro para especificar os materiais obturantes.

Visando contribuir no desenvolvimento de métodos que determinem a combinação ideal dos obturantes, este trabalho avaliou o uso da técnica de inversão com minimização de resíduos como ferramenta para a obtenção da granulometria ideal para a formação do reboco em um determinado reservatório.

\section{MATERIAIS E MÉTODOS}

A obtenção da proporção otimizada dos materiais obturantes pode ser feita a partir de métodos de combinação linear e procedimentos de inversão. Desta forma, assumindo uma relação linear entre duas funções: $g(\varepsilon)$ e $f(\varepsilon)$ tem-se:

$$
g(\varepsilon)=\sum_{i=1}^{N} f_{i}(\varepsilon) c_{i}
$$

em que $g(\varepsilon)$ é a função que representa a distribuição de partículas ideal, $f(\varepsilon)$ é a função que representa a distribuição granulométrica dos materiais obturantes, $\varepsilon$ é o diâmetro das partículas, $C_{i}$ indica a proporção de cada material na combinação.

De forma equivalente:

$$
G=F C
$$

A diferença entre a distribuição desejada $G$ e a distribuição resultante dos diferentes materiais $C F$ é denominada de resíduo $(R)$ e deve ser idealmente igual à zero:

$$
R=G-F C
$$

Aplicaremos aqui a metodologia do estimador de mínimos quadrados para encontrar o melhor conjunto para o vetor C. Este procedimento minimiza a norma absoluta $(Q)$, escrita a seguir:

$$
Q=\sum_{j}\left[g\left(\varepsilon_{j}\right)-\sum_{i} f_{i}\left(\varepsilon_{j}\right) c_{i}\right]^{2}
$$

Para minimizar a norma, aplicaremos o procedimento de minimização:

$$
\frac{\partial Q}{\partial c_{k}}=0
$$

que resulta em:

$$
0=\sum_{j}\left[g\left(\varepsilon_{j}\right)-\sum_{i} f_{i}\left(\varepsilon_{j}\right) c_{i}\right] f_{k}\left(\varepsilon_{j}\right)=\sum_{j} r_{j} f_{k}\left(\varepsilon_{j}\right)
$$

$\mathrm{Na}$ forma matricial a Equação (6) é escrita como:

$0=R^{T} F$

ou, na sua forma transposta:

$0=F^{T} R$

Substituindo a relação da Equação (3) na Equação (8) temos:

$0=F^{T}(G-F C)$

Com isso, encontra-se a relação que fornece a melhor estimativa para os valores de $C$ :

$$
C=\left(F^{T} F\right)^{-1} F^{T} G
$$


Os princípios desta metodologia são amplamente empregados em problemas de inversão em geofísica, aplicações da mesma podem ser encontradas Silva et al. (2010); Barbosa et al. (2011).

$\mathrm{O}$ procedimento descrito foi implementado para combinação de uma quantidade qualquer de materiais. Neste trabalho, a mistura dos materiais foi realizada a partir da combinação linear de três gaussianas idealizadas $(f(\varepsilon))$ como um estudo de caso. A distribuição de tamanho de partículas de um material pulverulento pode ser descrita de forma bastante satisfatória por $f(\varepsilon)$, o que justifica o seu uso neste estudo.

$\mathrm{Na}$ Figura 1 pode-se observar as três gaussianas. Tais funções representam a distribuição granulométrica de três materiais obturantes. As gaussianas são combinadas de forma a produzir com o mínimo de erro a distribuição granulométrica ideal (também idealizada para a realização deste estudo).

Figura1 - Curva de distribuição de frequência em função do diâmetro para os três materiais idealizados por este trabalho, juntamente com a curva da distribuição granulométrica ideal.

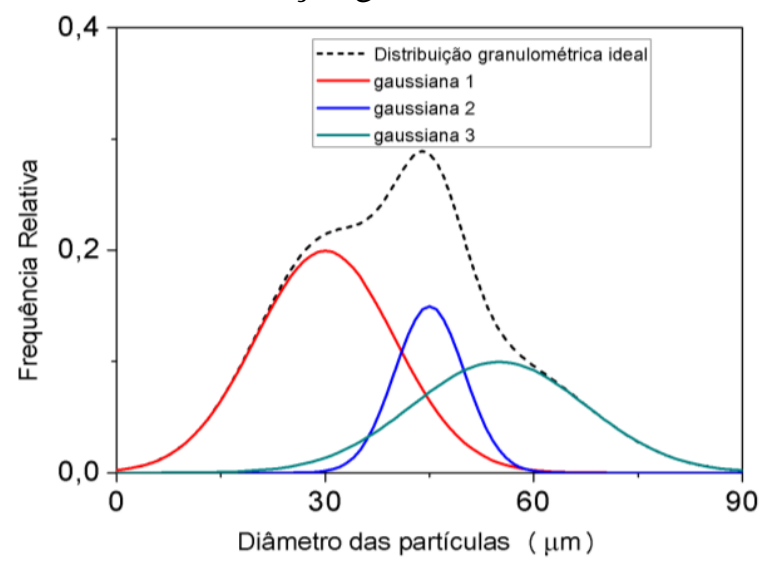

\section{RESULTADOS}

Os resultados da combinação entre as três gaussianas que forneceu a melhor aproximação em relação a distribuição desejada estão mostradas na Figura 2. A distribuição granulométrica desejada representou neste trabalho a melhor distribuição de tamanhos de partículas que forma um reboco fino e de baixa permeabilidade.

Figura2 - Distribuição de frequência em função do diâmetro das partículas obtida pela combinação de três gaussianas.

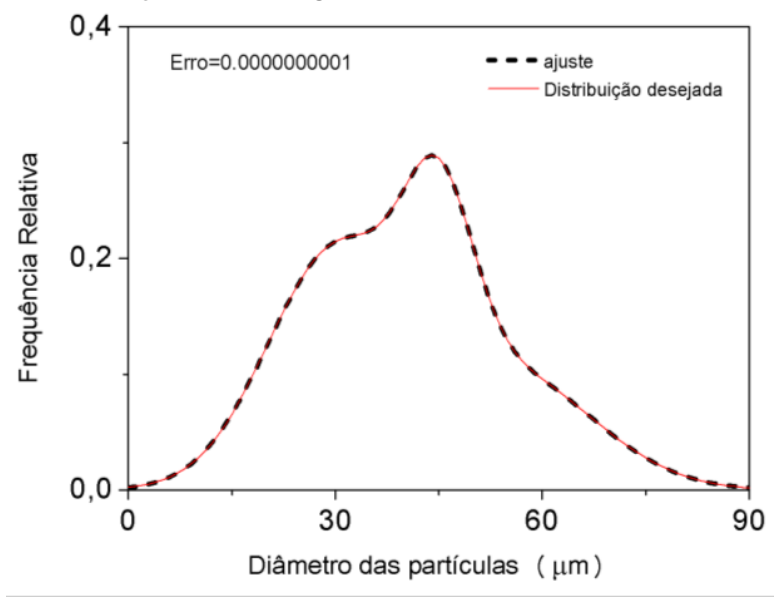

Observa-se na Figura 2, que os valores da combinação das três gaussianas aproximaram-se da distribuição desejada com um erro próximo de zero. Desta forma, podese concluir que o uso da técnica de inversão com minimização de resíduos mostrou-se altamente satisfatória.

As porcentagens em massa das gaussianas 1, 2 e 3 que combinadas geraram na Figura 2 a curva de ajuste foram respectivamente de $50 \%, 19 \%$ e $31 \%$. Também pode-se observar que a utilização de apenas três materiais foi suficiente para realizar a aproximação desejada.

$\mathrm{O}$ baixo valor do erro entre a distribuição de tamanhos desejada e a alcançada com a combinação das gaussianas foi conseguido após diversas iterações sucessivas. A redução do erro no desenvolvimento do processo iterativo está mostrada na Figura 3.

Verifica-se na Figura 3 que o código desenvolvido mostrou uma minimização dos erros de forma bastante rápida, confirmando a eficácia da metodologia utilizada para a 
resolução dos problemas tratados neste trabalho.

Figura 3 - Minimização do erro entre a distribuição de tamanhos desejada e a alcançada com a combinação das gaussianas.

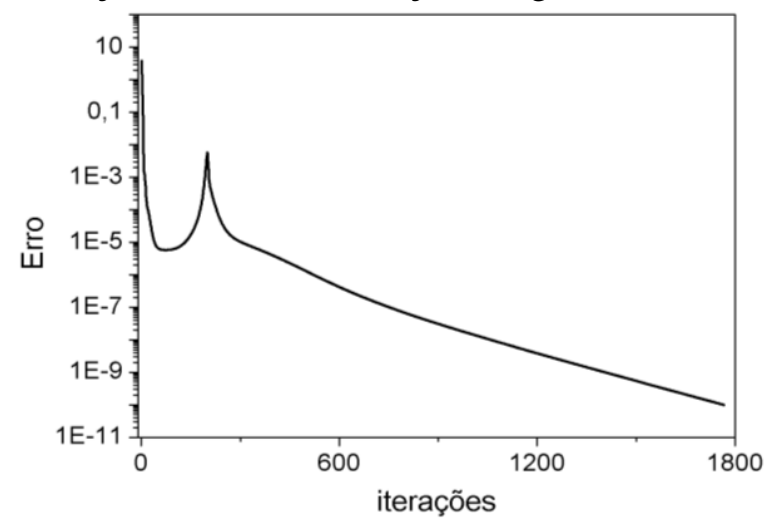

\section{CONCLUSÕES}

A técnica de inversão com minimização de resíduos como método para determinar a distribuição granulométrica ideal dos agentes obturantes, mostrou-se satisfatória. A partir da combinação de três materiais diferentes foi possível verificar um erro próximo de zero entre a curva ideal e a obtida com a combinação dos materiais.

A ferramenta desenvolvida neste trabalho pode ser utilizada também em campo para auxiliar na determinação da proporção em que diferentes materiais obturantes devem ser combinados para a formação de um reboco de baixa permeabilidade.

\section{REFERÊNCIAS}

ABRAMS, A. Mud Design to Mike Rock Impairment due to Particle Invasion. Journal of Petroleum Technology, p. 586-592, 1977.

BARBOSA, V. C. F.; SILVA, J. B. C.; VASCONCELOS, S. S. e OLIVEIRA, S. F. Entropic Regularization to Assist a Geologist in Producing a Geologic Map. Entropy, vol. 13, p. 790-804, 2011.
DICK, M. A.; HEINZ, T. J.; SVOBODA, C. F.; ASTON, M. Optimizing the Selection of Bridging Particles for Reservoir Drilling Fluids, International Symposium on Formation Damage, SPE 58793, Louisiana, EUA, 2000.

ENBAIA Dr, A. E.; RAMDZANI, I. A. B. A. Pore size geometry of reservoir rocks used as key factor for drilling and completion fluid design of oil wells. European Scientific Journal, p. 102-113, 2014.

KAEUFFER, M. Determination de L'Optiumum de Remplissage Granulometrique et Quelques Proprietes S'y Rattachant, In: Congress International de I'A.F.T.P.V., Anais... Rouen, 1973.

\section{MOREIRA, B. A. Estudo da sedimentação} em suspensões de fluidos com características reológicas pseudoplásticas. Tese (Doutorado em Engenharia Química), Universidade Federal de Uberlândia, Uberlândia, Brasil, 2014

SILVA, J. B. C.; VASCONCELOS, S. S.; BARBOSA, V. C. F. Apparent-magnetization mapping using entropic regularization. Geophysics, vol. 75, p. 39-50, 2010.

WALDMANN, A. T. A. Mecanismos que governam a efetividade de agentes obturantes no controle da invasão de fluidos de perfuração na rocha reservatório de petróleo. Dissertação (Mestrado em Engenharia Química) - PUC-RIO, Rio de Janeiro-RJ, Brasil, 2005.

WENQIANG, F; JIENIAN, Y. Designing Drill-in Fluids by Using Ideal Packing Technique, Petroleum Science, Vol. 4, n. 2, p. 44-51, 2007. 